\title{
Spatial distribution of perfluoroalkyl acids in surface sediments of the German Bight, North Sea
}

\author{
Zhen Zhao ${ }^{\text {a,b,c }}$, Zhiyong Xie ${ }^{\text {a,* }}$, Jianhui Tang ${ }^{\text {c,* }}$, Gan Zhang ${ }^{b}$, Ralf Ebinghaus ${ }^{\text {a }}$ \\ ${ }^{a}$ Helmholtz-Zentrum Geesthacht, Centre for Materials and Coastal Research, Institute of Coastal Research, Max-Plank Street 1, 21502 Geesthacht, Germany \\ b State Key Laboratory of Organic Geochemistry, Guangzhou Institute of Geochemistry, CAS, Kehua Road 511, Guangzhou 510631, China \\ c Yantai Institute of Coastal Zone Research, CAS, Chunhui Road 17, Yantai 264003, China
}

\section{H I G H L I G H T S}

- Declining PFOA and PFOS levels implied the effect of regulating C8-based products

- PFBA and PFBS occurred in the sediments of the German Bight

- PFOS in marine sediment may present a risk for benthic organisms in the German Bight

\section{A R T I C L E I N F O}

\section{Article history:}

Received 22 October 2014

Received in revised form 18 December 2014

Accepted 19 December 2014

Available online $\mathrm{xxxx}$

Editor: Adrian Covaci

\section{Keywords:}

Perfluoroalkyl acids

Perfluorooctane sulfonic acid

Surface sediment

German Bight

\begin{abstract}
A B S T R A C T
Perfluoroalkyl acids (PFAAs) have been determined in the environment globally. However, studies on the occurrence of PFAAs in marine sediment remain limited. In this study, 16 PFAAs are investigated in surface sediments from the German Bight, which provided a good overview of the spatial distribution. The concentrations of $\Sigma$ PFAAs ranged from 0.056 to $7.4 \mathrm{ng} / \mathrm{g}$ dry weight. The highest concentration was found at the estuary of the River Ems, which might be the result of local discharge source. Perfluorooctane sulfonic acid (PFOS) was the dominant compound, and the enrichment of PFOS in sediment might be strongly related to the compound structure itself. The geographical condition of the German Bight influenced the movement of water and sediment, resulting in complex distribution. Following normalization according to total organic carbon (TOC) content, PFAA distributions showed a different picture. Significant linear relationships were found between total PFAA concentrations and TOC $\left(\mathrm{R}^{2}=0.50, \mathrm{p}<0.01\right)$. Compared with a previous study conducted in the same area, a declining trend was presented for the concentrations of PFOS and perfluorooctanoic acid (PFOA). Compound structure, geographical condition, and organic carbon in the sediment influence the distribution of PFAAs in the German Bight. Environmental risk assessment indicated that the risk from PFOA is negligible, whereas PFOS in marine sediment may present a risk for benthic organisms in the German Bight.
\end{abstract}

(c) 2014 Elsevier B.V. All rights reserved.

\section{Introduction}

Perfluoroalkyl acids (PFAAs) have been produced and utilized worldwide in high volumes for several decades, in consumer products such as polymerization aids; stain repellents in carpets; textile, and leather and paper products; and constituents of firefighting foams and fluoropolymer manufacturing, owing to their unique physical and chemical properties of thermal stability, surfactant property, hydrophobicity, and oleophobicity (Giesy and Kannan, 2001; Lindstrom et al., 2011; Prevedouros et al., 2006). PFAAs can be directly discharged into the aquatic environment via wastewater treatment plants, and from production and application processes (Ahrens, 2011; Armitage et al.,

\footnotetext{
* Corresponding authors.

E-mail addresses: zhiyong.xie@hzg.de (Z. Xie), jhtang@yic.ac.cn (J. Tang).
}

2006; Sinclair and Kannan, 2006). They can be transformed from neutral poly-/per-fluoroalkyl substances (PFASs) via atmospheric oxidation and biotic degradation (Ellis et al., 2004; Martin et al., 2006; Tomy et al., 2004; Wallington et al., 2006). Neutral PFASs have been released to the environment in large quantities (Paul et al., 2009; Prevedouros et al., 2005) and are known to be long-range-transported pollutants (Ahrens et al., 2009a; Dreyer et al., 2009; Jahnke et al., 2007; Shoeib et al., 2006, 2010).

Previous studies of PFAAs have raised considerable concern regarding their global distribution (Ahrens et al., 2009a; Butt et al., 2010; Cai et al., 2012; Kannan, 2011; Taniyasu et al., 2013; Yamashita et al., 2005), bioaccumulation and/or biotransformation in organisms and marine mammals, environmental degradation and potential toxicity (Giesy et al., 2010; Naile et al., 2010). In recent years, PFAAs have been reported in different environmental compartments such as air (Dreyer 
et al., 2009; Li et al., 2011), water (Ahrens, 2011; Benskin et al., 2012), sediment (Bao et al., 2010; Higgins et al., 2005; Lam et al., 2014; Pan et al., 2014; Theobald et al., 2012; Zhao et al., 2013), and in biota (Giesy and Kannan, 2001; Houde et al., 2006; Kannan et al., 2006). Among the PFAAs, perfluorinated sulfonate acids (PFSAs) and perfluorinated carboxylic acids (PFCAs), e.g. PFOA and PFOS, have been well studied in aquatic environments, including their presence in the polar regions (Butt et al., 2010; Kwok et al., 2013). Because of their ubiquity and potential toxicity (Andersen et al., 2008; Hekster et al., 2003), the production and use of PFOS and PFOA are regulated worldwide. EU Directive 2006/122/EC required European countries to restrict the manufacture and use of PFOS from 2008 (EPC, 2006). National programs in the United States and Canada are attempting to regulate the use of PFOA, its salts and precursors (USEPA, 2006; Vierke et al., 2012). In 2009, PFOS and its salts, and perfluorooctanesulfonyl fluoride (PFOSF) were added to Annex B of the Stockholm Convention as new persistent organic pollutants.

PFAAs can be transported into coastal and marine environments via both riverine and atmospheric pathways. The sediment is highly important for the evaluation of their accumulation in coastal basins and potential impacts on benthic organisms. Several studies have reported PFAAs in sediment from China (Bao et al., 2009, 2010, 2012; Pan et al., 2014; Pan and You, 2010; Zhao et al., 2013), Japan (Ahrens et al., 2011), the United States (Senthil et al., 2009), and Europe (Becker et al., 2008; Beškoski et al., 2013; Campo et al., 2015; Hloušková et al., 2014; Labadie and Chevreuil, 2011a,b; Perra et al., 2013; Theobald et al., 2012). PFOS and PFOA were found as dominant homologues in PFAAs in most studies. Higgins and Luthy (2006) reported that the sorption of PFAAs onto sediment was influenced by organic carbon content, $\mathrm{pH}$, and electrolytes in the solution, and by the compounds' structure.

The German Bight is the southeastern Bight of the North Sea, which receives large volumes of water containing particulate matter from the Rhine $\left(2260 \mathrm{~m}^{3} / \mathrm{s}\right)$, Ems $\left(85 \mathrm{~m}^{3} / \mathrm{s}\right)$, Weser $\left(350 \mathrm{~m}^{3} / \mathrm{s}\right)$ and Elbe $\left(750 \mathrm{~m}^{3} / \mathrm{s}\right.$ ) rivers (Kampa et al., 2003). PFAAs have been frequently detected in seawater of the German Bight and its tributaries. The annual fluxes of PFASs in the North Sea were estimated to be $335 \pm 100 \mathrm{~kg} /$ year from the River Elbe and $102 \pm 22 \mathrm{~kg} /$ year from the River Weser (Zhao et al., 2014). PFAAs were also identified in sediment collected from the German Bight in 2004 and 2005 (Theobald et al., 2012). However, there is less information on the latest status of PFAAs in sediment of the German Bight compared with that in seawater.

In this study, we collected sediment samples in the German Bight including the estuaries of the rivers Ems, Weser, and Elbe (Fig. 1). The distribution of PFAAs was discussed owing to the estuarine and offshore sampling program and total organic carbon content. The obtained PFAA data are compared with previous studies to evaluate the effect of regulatory controls. Furthermore, the potential risks of PFOS and PFOA in the aquatic ecosystem were assessed for the German Bight.

\section{Materials and methods}

\subsection{Chemicals and standards}

The native standards mixture including 16 PFAAs (i.e. $\mathrm{C}_{4^{-}}, \mathrm{C}_{6^{-}}, \mathrm{C}_{8^{-}}$ and $\mathrm{C}_{10^{-}}$PFSAs and $\mathrm{C}_{4^{-}}, \mathrm{C}_{14^{-}}$-PFCAs, $>99 \%$ ) was purchased from Wellington Laboratories. The mass-labeled standards were obtained from the same company, which contained 9 compounds (i.e. ${ }^{13} \mathrm{C}$ labeled $\mathrm{C}_{4^{-}}, \mathrm{C}_{6^{-}}, \mathrm{C}_{8^{-}}, \mathrm{C}_{12^{-}}$PFCAs, $\mathrm{C}_{8}$-PFSAs, and perfluorooctane sulfonamide (FOSA) and ${ }^{18} \mathrm{O}$-labeled $\mathrm{C}_{6}$-PFSAs, $>99 \%$ ). Methanol (Suprasolv grade), ammonium hydroxide (25\%, Suprapur), sodium hydroxide, and hydrochloric acid were purchased from Merck (Darmstadt, Germany). Millipore water was produced by a Milli-Q Plus 185 system (Zug, Germany).

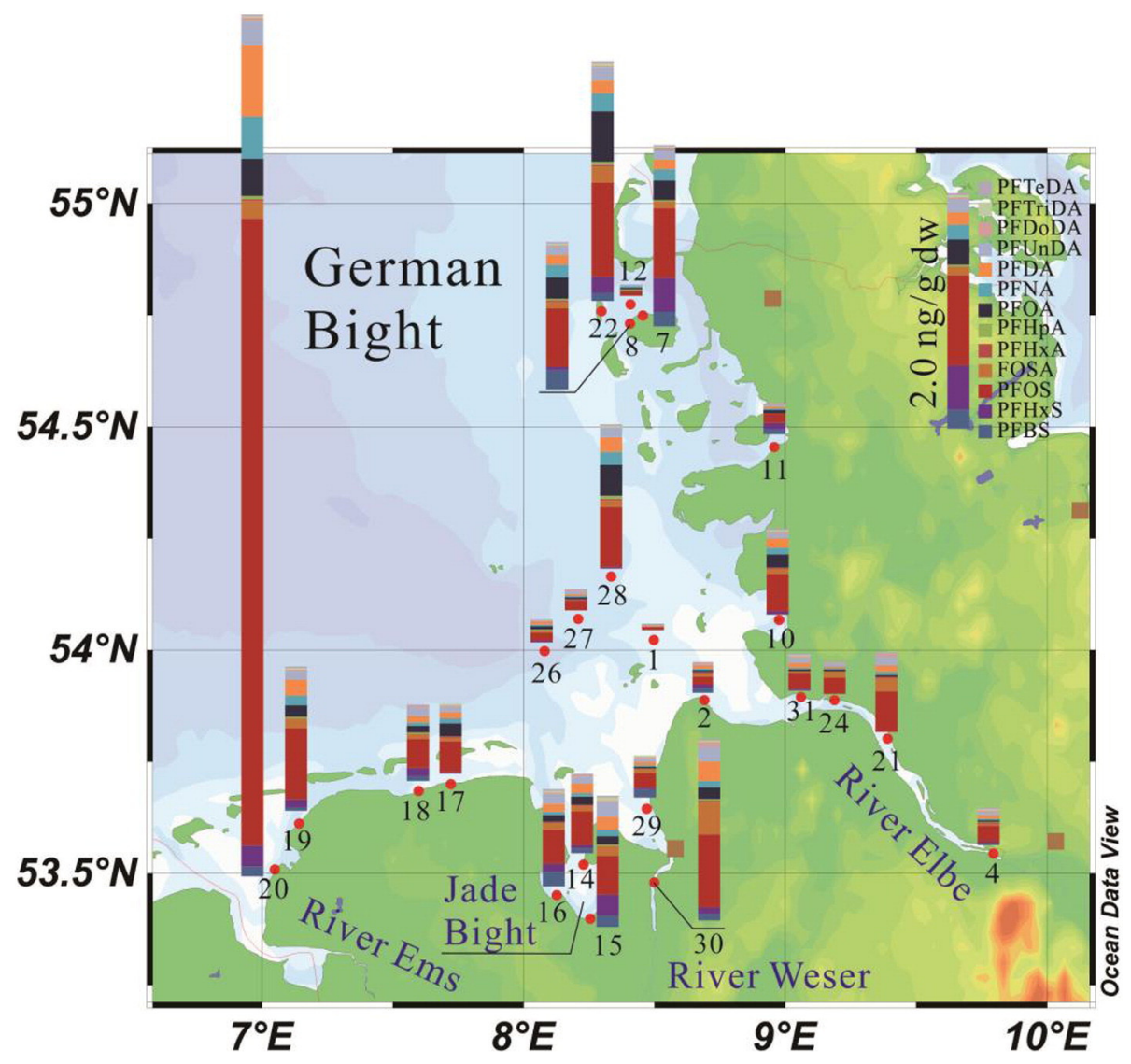

Fig. 1. Concentrations (ng/g dw) and spatial distribution of PFAAs in German Bight surface sediment. 


\subsection{Sampling and extraction}

From February to May 2011, 24 surface sediment samples were collected at the German Bight coast (Fig. 1). In the region where sediment emerged during low tide, samples were collected by stainless steel spoon, and where sediment could not be reached by hiking, samples were obtained by a grab during cruise aboard the research vessels Ludwig Prandtl, and Heincke. The top 0-5 cm layer of sediment was sealed in aluminum boxes, frozen and stored at $-20{ }^{\circ} \mathrm{C}$ until analysis. The aluminum boxes were rinsed using methanol twice before being used. Sediments were freeze-dried (Martin Christ GAMMA 1-20) for $72 \mathrm{~h}$, homogenized by agate mortar and pestle and sealed in methanol-rinsed brown jars until extraction.

The extraction and clean-up methods were modified from those reported by Higgins et al. (2005) and were described elsewhere (Zhao et al., 2013). Briefly, $8 \mathrm{~g}$ sediments were transferred to $50 \mathrm{~mL}$ methanol-rinsed polypropylene (PP) tubes and spiked with masslabeled standard mixture (1 $\mathrm{ng}$ ). $10 \mathrm{~mL}$ methanol was added and the mixture was soaked for $30 \mathrm{~min}$ and centrifuged for $8 \mathrm{~min}$ at $3000 \mathrm{rpm}$. The supernatant was collected in a methanol-rinsed glass flask. Sonication was repeated three times, yielding a total of $30 \mathrm{~mL}$ supernatant. The combined supernatant was concentrated to $3 \mathrm{~mL}$ by a rotary evaporator for clean-up.

An ENVI-Carb tube ( $3 \mathrm{~mL}, 0.25 \mathrm{~g}$ ) was employed for clean-up. All the cartridges were washed by $10 \mathrm{~mL}$ methanol prior to use. After spiking the sample, $10 \mathrm{~mL} 0.1 \% \mathrm{NH}_{4} \mathrm{OH}$ methanol was added and the eluent was collected in glass tubes, before being further concentrated to $150 \mu \mathrm{L}$ under gentle nitrogen stream. All samples were spiked with 1 ng mass-labeled 8:2 fluorotelomer unsaturated acid (8:2 FTUCA) as injection standard prior to analysis.

\subsection{Instrument analysis}

The instrument analysis was performed using a high-performance liquid chromatography electrospray ionization-tandem mass spectrometry system (HPLC-ESI-MS/MS) operating in negative ionization mode that consisted of an HP 1100 HPLC system (Agilent Technologies) coupled to an API 3000 triple-quadrupole mass spectrometer (Applied Biosystems/MDS SCIEX). All Teflon parts were replaced by polypropylene parts to avoid possible contamination. The separation column was a Synergi Hydro RP 80A column $(150 \times 2 \mathrm{~mm}, 4 \mu \mathrm{m})$ from Phenomenex, which was combined with a suitable guard column: Synergi $2 \mu$ Hydro RP Mercury $(20 \times 2 \mathrm{~mm}, 2 \mu \mathrm{m})$. The mobile phases employed were Millipore water and methanol both containing with $10 \mathrm{mmol} / \mathrm{L}$ ammonium acetate. The gradient of the mobile phase started with $30 \%$ methanol and then increased to $70 \%$ methanol over 3 min. After continuously increasing to $100 \%$ methanol over $28 \mathrm{~min}$, the gradient was held for $7 \mathrm{~min}$. The acquisition time was $40 \mathrm{~min}$ with 7 min equilibration. An API 3000 mass spectrometer was operated in multiple reaction monitoring (MRM) mode. Details of the instrument parameters, precursors, and product ions were described and listed in Ahrens et al. (2009a) and Zhao et al. (2013). Total organic carbon (TOC) was determined via an elemental analyzer (LECO RC 612).

\subsection{Quality assurance and quality control}

All the standards were checked before use, to avoid possible contamination from storage. No contamination was found in the standard mixtures. Chemicals (i.e. methanol, ammonium hydroxide, sodium hydroxide, and hydrochloric acid) were all examined, and no PFAAs were detectable. The blank ENVI-Carb cartridges were eluted and no PFAAs were found. One procedural blank was analyzed for every 8 samples. PFOS and FOSA were detected in procedural blanks at concentrations of $2 \pm 0.5 \mathrm{pg} / \mathrm{g}$ and $3 \pm 1 \mathrm{pg} / \mathrm{g}$, respectively.

The instrumental detection limit (IDL) was determined using a signal-to-noise ratio of 3 at the lowest level $(0.2 \mathrm{ng} / \mathrm{mL})$ in the calibration. IDLs varied from $2 \mathrm{pg}$ (perfluorohexane sulfonate acid, PFHxS) to $16 \mathrm{pg}$ (perfluorobutanoic acid, PFBA). The method detection limit (MDL) was determined in two ways: 1) for the target analytes that were not detected in procedural blanks, MDLs were determined using a signal-to-noise ratio of 10 , and MDL varied from $1 \mathrm{pg} / \mathrm{g}$ dry weight (dw, PFHxS) to $11 \mathrm{pg} / \mathrm{g}$ dw (PFBA); 2) for PFOS and FOSA, MDLs were extrapolated under $98 \%$ confidence intervals using the standard deviations and values in the blanks, giving MDLs for PFOS and FOSA of $3 \mathrm{pg} / \mathrm{g}$ dw and $8 \mathrm{pg} / \mathrm{g} \mathrm{dw}$, respectively.

To determine the precision of the method, sediments from the Arctic Ocean were employed. First, Arctic sediments from three sampling sites were extracted and analyzed, and no PFAAs were detectable. The PFAAfree sediments were spiked with PFAA standards. Sediments were then extracted, cleaned-up and analyzed, and recoveries of PFAAs were determined from $52 \%$ (PFBA) to $125 \%$ (PFUnDA) with mean value of $87 \% \pm 16 \%$.

Recoveries for German Bight sediments were calculated using masslabeled standards. The values ranged from $61 \pm 11 \%\left({ }^{13} \mathrm{C}_{8} \mathrm{FOSA}\right)$ to $106 \pm 13 \%\left({ }^{13} \mathrm{C}_{4} \mathrm{PFBA}\right)$. In each extraction series ( 8 samples), one parallel sample was added, and the deviations of target analytes were within $25 \%$.

\section{Results and discussion}

\subsection{Distribution of PFAAs in German Bight surface sediment}

All 16 of the PFAAs (PFBA is exclude from discussion) were detected in German Bight surface sediment. Concentrations of individual PFAAs and $\Sigma$ PFAAs are listed in Table 1. Concentrations of $\Sigma$ PFAAs ranged from 0.056 to $7.4 \mathrm{ng} / \mathrm{g} \mathrm{dw}$ (mean $1.0 \mathrm{ng} / \mathrm{g} \mathrm{dw}$ ). ¿PFSA concentrations ranged from 0.023 to $5.7 \mathrm{ng} / \mathrm{g} \mathrm{dw}$ (mean $0.61 \mathrm{ng} / \mathrm{g} \mathrm{dw}$ ), which were higher than those of $\Sigma$ PFCAs $(0.032-1.6 \mathrm{ng} / \mathrm{g} \mathrm{dw}$, mean $0.34 \mathrm{ng} / \mathrm{g} \mathrm{dw})$. PFOS was the dominant compound (range 0.023-5.4 ng/g dw, mean $0.27 \mathrm{ng} / \mathrm{g} \mathrm{dw}$ ), accounting for $24-72 \%$ of $\Sigma$ PFAA concentration. With the exception of PFOS, all the other PFSAs had average compositions less than $8 \%$. PFOA and PFDA were the dominant PFCAs, each accounting for $10 \%$ ¿PFAA. The average concentrations of longer-chained PFCAs, i.e. PFUnDA, PFDoDA and perfluorotridecanoic acid (PFTriDA), were 0.059, 0.015 , and $0.014 \mathrm{ng} / \mathrm{g} \mathrm{dw}$, respectively, whereas, shorter-chained PFCAs i.e. perfluoropentanoic acid (PFPeA), perfluorohexanoic acid (PFHxA) and perfluoroheptanoic acid (PFHpA), exhibited higher concentrations of $0.05,0.07$, and $0.08 \mathrm{ng} / \mathrm{g} \mathrm{dw}$, respectively. Ahrens et al. (2009b) reported a different situation for PFAA concentrations in German Bight surface water in 2007. In water phase, perfluorobutane sulfonate acid (PFBS) was the dominant compound (maximum concentration $17.7 \mathrm{ng} / \mathrm{L}$ ), and among PFCAs, shorter-chained PFAAs had greater contribution than longer-chained PFAAs. That means the composition of PFAAs in sediment highly related to that in water. Higgins and Luthy (2006) revealed the relationship between the structural features of compounds and the sorption of PFAAs onto sediments. PFAAs with sulfonate heads that showed stronger affinity than those of carboxylates, and longer-chained compounds had stronger affinity than shorterchained ones, as a result of more $\mathrm{CF}_{2}$ moieties in their chemical structure. The significant differences between PFAA profiles in water and in sediments from German Bight suggested that the compound properties strongly influenced the distribution of PFAAs.

The spatial distribution of PFAAs is presented in Fig. 1. The highest concentration of $\Sigma$ PFAAs was detected at the estuary of the River Ems (site 20, $7.4 \mathrm{ng} / \mathrm{g} \mathrm{dw}$ ), which was more than ten times higher than that from the River Weser estuary (site 29, $0.47 \mathrm{ng} / \mathrm{g} \mathrm{dw}$ ) and twenty to thirty times higher than that from the River Elbe Estuary (sites 2 and 31, 0.26-0.31 ng/g dw). The River Ems has smaller discharge volume than the Weser and Elbe, and the cities along the River Ems are much smaller than Bremen (River Weser) and Hamburg (River Elbe) (Ahrens et al., 2009b). The domestic sources may not be the major contributors of PFAAs. Southwest of the River Ems estuary, the River Rhine discharges heavily contaminated water to the North Sea (Möller et al., 
Table 1

Concentrations (ng/g dry weight) of individual PFAAs and ZPFAAs in surface sediment of German Bight and the TOC content (\%).

\begin{tabular}{|c|c|c|c|c|c|c|c|c|c|c|c|c|c|c|c|c|c|c|c|c|}
\hline Site & TOC & PFBS & PFHxS & PFHpS & PFOS & PFDS & FOSA & PFPeA & PFHxA & PFHpA & PFOA & PFNA & PFDA & PFUnDA & PFDoDA & PFTriDA & PFTeDA & ¿PFSAs & ¿PFCAs & ¿PFAAs \\
\hline GB1 & 0.023 & $<0.006$ & $<0.001$ & $<0.01$ & 0.023 & $<0.006$ & $<0.008$ & $<0.003$ & $<0.001$ & $<0.001$ & 0.007 & 0.003 & 0.016 & 0.007 & $<0.003$ & $<0.005$ & $<0.003$ & 0.023 & 0.032 & 0.056 \\
\hline GB2 & 0.544 & 0.040 & 0.028 & $<0.01$ & 0.069 & $<0.006$ & 0.035 & $<0.003$ & 0.004 & 0.002 & 0.019 & 0.017 & 0.028 & 0.013 & 0.007 & $<0.005$ & $<0.003$ & 0.14 & 0.090 & 0.26 \\
\hline GB4 & 0.534 & 0.011 & 0.012 & $<0.01$ & 0.14 & $<0.006$ & 0.018 & $<0.003$ & 0.004 & 0.002 & 0.008 & 0.006 & 0.031 & 0.031 & 0.018 & 0.008 & 0.005 & 0.17 & 0.11 & 0.30 \\
\hline GB7 & 3.02 & 0.12 & 0.30 & 0.022 & 0.59 & $<0.006$ & 0.051 & $<0.003$ & 0.005 & 0.010 & 0.17 & 0.094 & 0.092 & 0.073 & 0.016 & 0.022 & 0.007 & 1.0 & 0.49 & 1.6 \\
\hline GB8 & 3.75 & 0.17 & 0.035 & 0.014 & 0.49 & $<0.006$ & 0.059 & $<0.003$ & 0.008 & 0.017 & 0.19 & 0.10 & 0.088 & 0.063 & 0.015 & 0.018 & 0.005 & 0.71 & 0.50 & 1.3 \\
\hline GB10 & 1.17 & 0.006 & 0.018 & $<0.01$ & 0.33 & $<0.006$ & 0.039 & $<0.003$ & 0.009 & 0.008 & 0.10 & 0.059 & 0.081 & 0.050 & 0.013 & 0.009 & 0.004 & 0.35 & 0.34 & 0.73 \\
\hline GB11 & 0.370 & 0.042 & 0.058 & $<0.01$ & 0.078 & $<0.006$ & $<0.008$ & $<0.003$ & 0.003 & 0.001 & 0.022 & 0.013 & 0.017 & 0.016 & 0.003 & $<0.005$ & $<0.003$ & 0.18 & 0.076 & 0.25 \\
\hline GB12 & 0.150 & $<0.006$ & 0.002 & $<0.01$ & 0.035 & $<0.006$ & 0.009 & 0.005 & 0.005 & 0.003 & 0.017 & 0.013 & 0.009 & 0.010 & $<0.003$ & $<0.005$ & $<0.003$ & 0.037 & 0.060 & 0.11 \\
\hline GB14 & 1.67 & 0.037 & 0.038 & $<0.01$ & 0.29 & $<0.006$ & 0.043 & $<0.003$ & 0.005 & 0.005 & 0.063 & 0.037 & 0.074 & 0.058 & 0.011 & 0.011 & 0.003 & 0.37 & 0.27 & 0.68 \\
\hline GB15 & 4.68 & 0.10 & 0.17 & 0.011 & 0.34 & $<0.006$ & 0.070 & $<0.003$ & 0.006 & 0.010 & 0.077 & 0.050 & 0.12 & 0.11 & 0.024 & 0.022 & 0.008 & 0.63 & 0.43 & 1.1 \\
\hline GB16 & 1.19 & 0.12 & 0.074 & $<0.01$ & 0.29 & $<0.006$ & 0.056 & $<0.003$ & 0.007 & 0.004 & 0.059 & 0.031 & 0.064 & 0.077 & 0.016 & 0.015 & 0.004 & 0.49 & 0.28 & 0.82 \\
\hline GB17 & 0.625 & $<0.006$ & 0.022 & $<0.01$ & 0.25 & $<0.006$ & 0.031 & 0.003 & 0.011 & 0.010 & 0.10 & 0.041 & 0.059 & 0.046 & 0.010 & 0.010 & $<0.003$ & 0.27 & 0.29 & 0.60 \\
\hline GB18 & 1.28 & 0.042 & 0.066 & $<0.01$ & 0.25 & $<0.006$ & 0.045 & $<0.003$ & 0.004 & 0.004 & 0.056 & 0.033 & 0.060 & 0.060 & 0.012 & 0.011 & 0.005 & 0.36 & 0.24 & 0.65 \\
\hline GB19 & 1.24 & 0.033 & 0.067 & $<0.01$ & 0.61 & $<0.006$ & 0.074 & $<0.003$ & 0.008 & 0.007 & 0.11 & 0.086 & 0.13 & 0.078 & 0.012 & 0.011 & 0.004 & 0.71 & 0.44 & 1.2 \\
\hline GB20 & 4.10 & 0.091 & 0.18 & 0.044 & 5.4 & $<0.006$ & 0.15 & 0.005 & 0.019 & 0.017 & 0.33 & 0.35 & 0.61 & 0.21 & 0.028 & 0.018 & 0.007 & 5.7 & 1.6 & 7.5 \\
\hline GB21 & 0.993 & $<0.006$ & 0.007 & $<0.01$ & 0.35 & $<0.006$ & 0.11 & $<0.003$ & 0.007 & 0.002 & 0.021 & 0.016 & 0.055 & 0.072 & 0.024 & 0.010 & 0.008 & 0.35 & 0.22 & 0.68 \\
\hline GB22 & 4.53 & 0.067 & 0.15 & $<0.01$ & 0.80 & $<0.006$ & 0.13 & $<0.003$ & 0.016 & 0.031 & 0.43 & 0.15 & 0.12 & 0.10 & 0.021 & 0.023 & 0.007 & 1.0 & 0.90 & 2.0 \\
\hline GB24 & 3.93 & $<0.006$ & 0.004 & $<0.01$ & 0.13 & $<0.006$ & 0.051 & $<0.003$ & 0.005 & 0.002 & 0.014 & 0.009 & 0.024 & 0.020 & 0.005 & $<0.005$ & $<0.003$ & 0.14 & 0.079 & 0.27 \\
\hline GB26 & 0.169 & $<0.006$ & 0.012 & $<0.01$ & 0.068 & $<0.006$ & 0.016 & 0.004 & 0.006 & 0.004 & 0.037 & 0.018 & 0.019 & 0.015 & $<0.003$ & $<0.005$ & $<0.003$ & 0.080 & 0.10 & 0.20 \\
\hline GB27 & 0.267 & $<0.006$ & $<0.001$ & $<0.01$ & 0.081 & $<0.006$ & 0.010 & $<0.003$ & 0.002 & $<0.001$ & 0.018 & 0.014 & 0.016 & 0.021 & 0.003 & $<0.005$ & $<0.003$ & 0.081 & 0.075 & 0.17 \\
\hline GB28 & 0.920 & $<0.006$ & 0.015 & $<0.01$ & 0.51 & $<0.006$ & 0.057 & 0.004 & 0.014 & 0.021 & 0.27 & 0.12 & 0.13 & 0.072 & 0.012 & 0.009 & 0.003 & 0.53 & 0.65 & 1.2 \\
\hline GB29 & 0.479 & 0.070 & 0.022 & 0.11 & 0.11 & $<0.006$ & 0.042 & $<0.003$ & 0.002 & 0.002 & 0.019 & 0.011 & 0.032 & 0.026 & 0.011 & 0.008 & $<0.003$ & 0.32 & 0.11 & 0.47 \\
\hline GB30 & 3.88 & 0.054 & 0.056 & $<0.01$ & 0.62 & 0.014 & 0.28 & 0.007 & 0.018 & 0.009 & 0.091 & 0.065 & 0.16 & 0.13 & 0.040 & 0.024 & 0.009 & 0.75 & 0.55 & 1.6 \\
\hline GB31 & 1.83 & $<0.006$ & 0.011 & $<0.006$ & 0.14 & $<0.006$ & 0.008 & $<0.003$ & 0.005 & 0.002 & 0.015 & 0.009 & 0.040 & 0.044 & 0.017 & 0.008 & 0.004 & 0.15 & 0.14 & 0.31 \\
\hline Max & 4.68 & 0.17 & 0.30 & 0.11 & 5.4 & 0.014 & 0.28 & 0.007 & 0.019 & 0.031 & 0.43 & 0.35 & 0.61 & 0.21 & 0.040 & 0.024 & 0.009 & 5.7 & 1.6 & 7.5 \\
\hline Min & 0.023 & 0.006 & 0.002 & 0.011 & 0.023 & 0.014 & 0.008 & 0.003 & 0.002 & 0.001 & 0.007 & 0.003 & 0.009 & 0.007 & 0.003 & 0.008 & 0.003 & 0.023 & 0.032 & 0.056 \\
\hline Mean & 1.72 & 0.067 & 0.061 & 0.041 & 0.50 & 0.014 & 0.063 & 0.005 & 0.007 & 0.008 & 0.093 & 0.056 & 0.086 & 0.059 & 0.015 & 0.014 & 0.006 & 0.61 & 0.34 & 1.0 \\
\hline Median & 1.18 & 0.054 & 0.031 & 0.022 & 0.27 & 0.014 & 0.048 & 0.004 & 0.006 & 0.004 & 0.057 & 0.032 & 0.059 & 0.054 & 0.013 & 0.011 & 0.005 & 0.35 & 0.26 & 0.66 \\
\hline
\end{tabular}


2010). North Sea currents have the potential to transport pollutants from the River Rhine estuary and other areas of the North Sea to German Bight (Ahrens et al., 2009c). The high concentrations of PFAAs detected at the River Ems estuary might be attributed to their transportation from other areas of the North Sea. Relatively high IPFAA concentrations were found at the northeast coast of German Bight, e.g., sites 7, 8, and 22 $(1.3-2.0 \mathrm{ng} / \mathrm{g} \mathrm{dw})$. These sampling sites were located at the inner area of the coast where the water exchange was relatively weak, which influenced the diffusion of contaminants. In the semi-enclosed Jade Bight (sites 14,15 , and $16,0.68-1.2 \mathrm{ng} / \mathrm{g} \mathrm{dw}$ ), ¿PFAA concentrations were

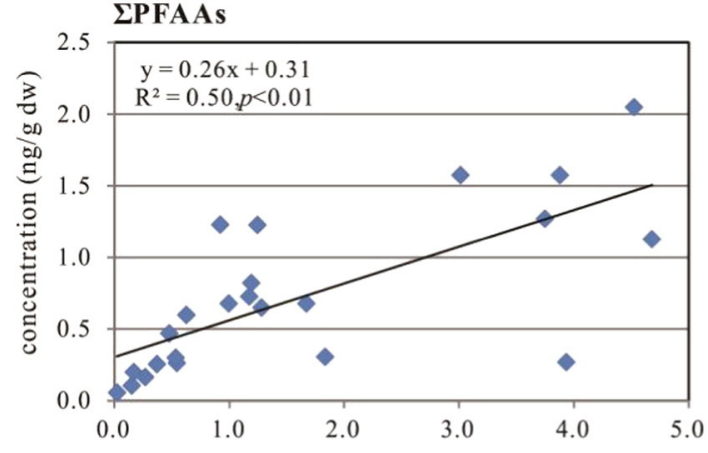

IPFSAs

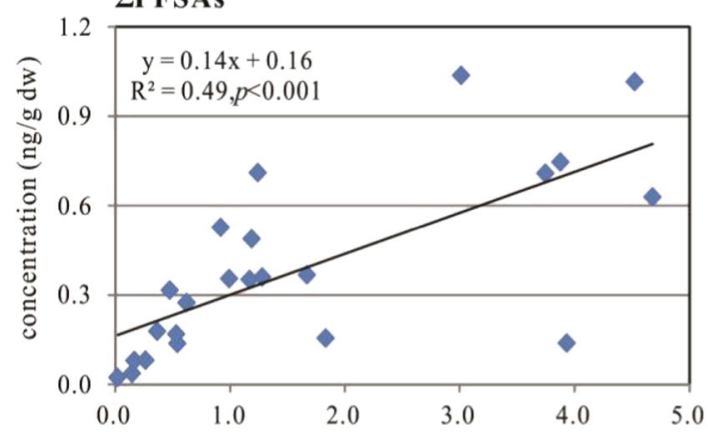

PFOA

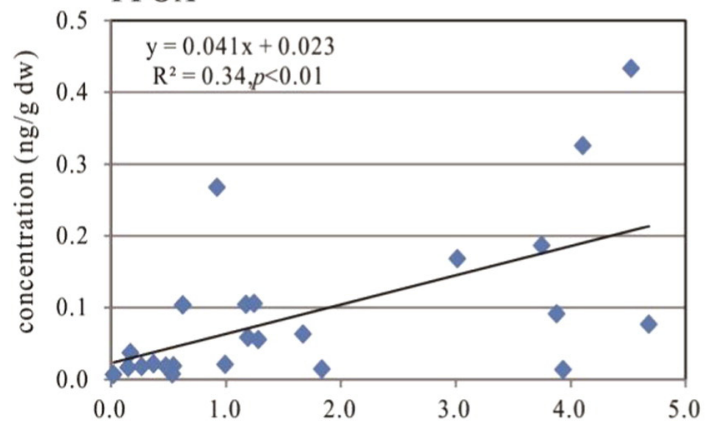

PFUnDA

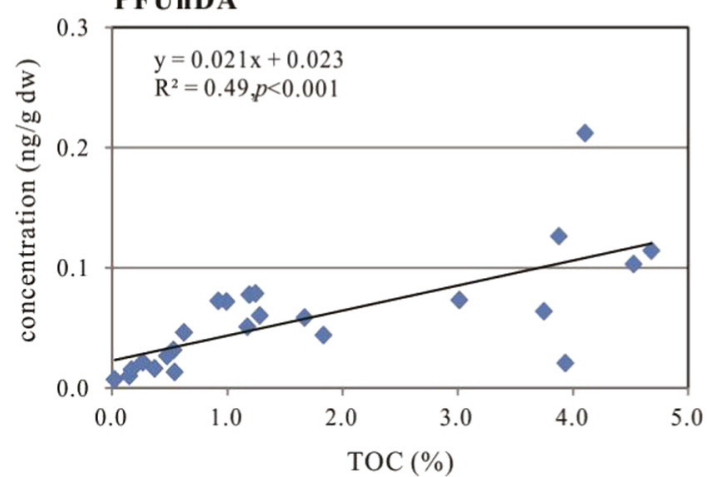

relatively stable and higher than those detected at the Weser and Elbe estuaries. Considering the difficulty of dilution and diffusion, PFAAs easily accumulated to relatively high levels in Jade Bight even though the sources were not highly concentrated. The sources were not very strong. Low $\Sigma$ PFAA concentrations were found at open areas of German Bight (sites 1, 26, and 27, 0.056-0.20 ng/g dw). The PFAA concentrations in water were reported to be relatively low in open areas (Ahrens et al., 2009b) and the suspended particles had to be transported long distances to deposit. The spatial distribution of PFAAs was related to the geography in German Bight.

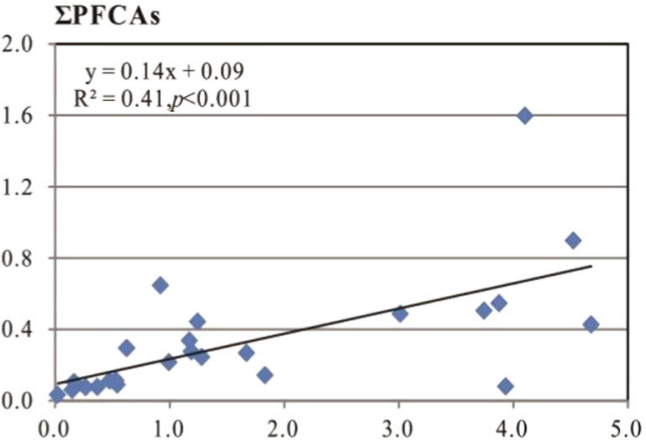

PFOS

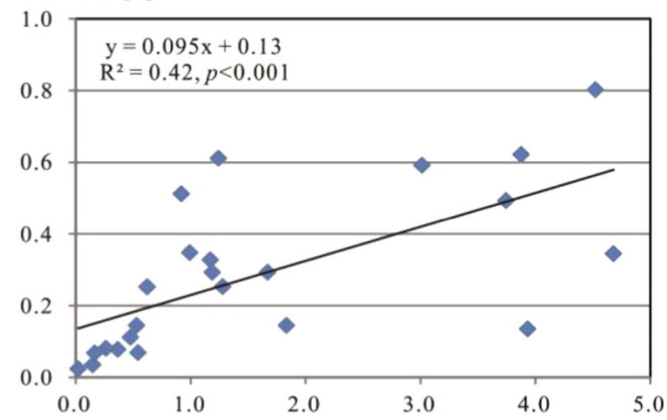

PFDA

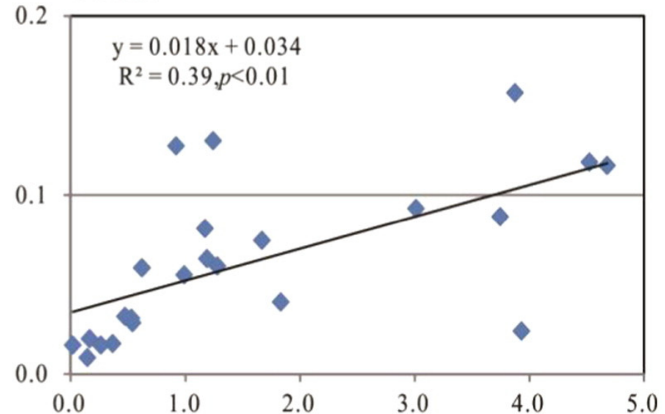

PFTriDA

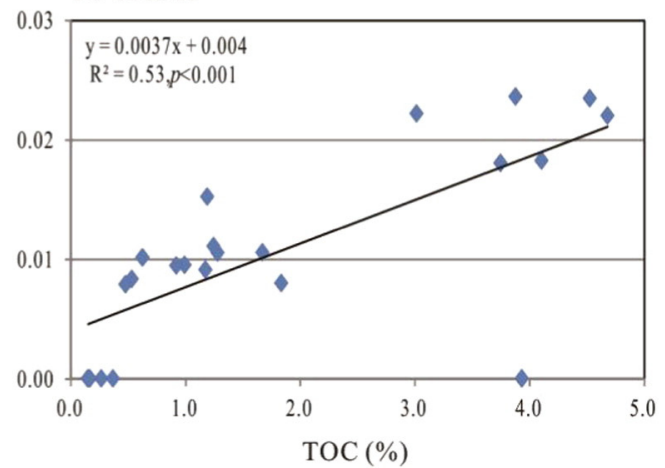

Fig. 2. Relationships between TOC content (\%) and PFAA concentrations (ng/g dw). 
The relationship between TOC contents and PFAA/乏PFAA concentrations was studied in German Bight sediment (see Fig. 2). A significant relationship was found between TOC and PFAA concentrations $\left(\mathrm{R}^{2}=0.50, p<0.01\right)$ without considering the highest concentration. The regression for $\Sigma$ PFCAs $\left(R^{2}=0.49, p<0.001\right)$ showed comparable relationship with that for $\Sigma$ PFSAs $\left(R^{2}=0.41, p<0.001\right)$. For individual PFAAs, PFOS concentrations (except the highest one) were significantly related to TOC content $\left(R^{2}=0.42, p<0.001\right)$. Longer-chained PFCAs, i.e. PFUnDA and PFTriDA, showed strong correlation to TOC content than PFOS $\left(R^{2}=0.49, p<0.001\right.$ for PFUnDA and $R^{2}=0.53, p<0.001$ for PFTriDA). PFOA and PFDA were less strongly correlated with TOC than were longer-chained PFCAs $\left(\mathrm{R}^{2}=0.34, p<0.01\right.$ for PFOA and $\mathrm{R}^{2}=$ $0.39, p<0.01$ for PFDA). The positive relationship between TOC and PFAA concentration was consistent with the discussion about sorption theory in Higgins and Luthy (2006), and the sorption affinity strongly related to the structure of the compounds. To reduce the influence of organic carbon, the concentrations were normalized by TOC content and the distributions are shown in Fig. 3. The highest value $(2.42 \mathrm{ng} / \mathrm{g}$ TOC) was found at sampling site 1 , which had the lowest TOC content. Site 20 showed the second-highest TOC of $1.81 \mathrm{ng} / \mathrm{g}$ and the highest TOC content. At the open offshore areas, e.g. sites 1, 26, 27, and 28, the values were significantly elevated, while at the inner areas, e.g. sites $20,15,30$, and 4 , the values were reduced. These findings indicate that, at offshore areas where sediments were homogenized, TOC strongly influenced the sorption of PFAAs.

\subsection{Comparison with previous studies}

In most previous studies (see Table 2), PFOS and PFOA were the predominant compounds detected in sediments, and PFOS usually presented higher concentration than PFOA. PFOS concentrations in German Bight in 2011 were comparable with those detected at Sydney Harbor, Australia (0.80-6.2 ng/g dw) (Thompson et al., 2011). Previous studies of coastal China, Japan, and USA reported lower PFOS concentrations than those recorded here for German Bight. Becker et al. (2008) reported lower PFOS concentrations $(0.07-0.31 \mathrm{ng} / \mathrm{g} \mathrm{dw})$ in surface sediments of the Red Main River in Germany than those found in this study. The PFOA concentrations in German Bight were lower than those reported in Laizhou Bay, China (0.07-1.8 ng/g dw) (Zhao et al., 2013) and Ariake Sea, Japan (0.84-1.1 ng/g dw) (Nakata et al., 2006). Comparable PFOA concentrations were reported for the North Bohai coast, China (nd-0.542 ng/g dw) (Wang et al., 2011), Tokyo Bay, Japan (0.45 ng/g dw) (Zushi et al., 2010), San Francisco coast, USA (nd$0.625 \mathrm{ng} / \mathrm{g} \mathrm{dw}$ ) (Higgins et al., 2005), and western Baltic Sea, Germany (0.06-0.68 ng/g dw) (Theobald et al., 2012).

Theobald et al. (2012) investigated PFAAs in sediment from German Bight in 2004. PFOS and PFOA were dominant, and PFNA and PFDA were detectable at most sites. Excluding the highest concentration in 2011, PFOS presented a declining trend from $2004(0.04-2.4 \mathrm{ng} / \mathrm{g} \mathrm{dw})$ to 2011 (0.023-0.80 ng/g dw). Germany ceased production of PFOS in 2007, after which PFOS was only used in some non-substitutable applications, e.g. metal plating, photographic applications and semiconductors (Carroll et al., 2009). The decreasing release from former sources might be the major reason for the observed lower concentrations, since PFOS is relatively stable in the environment. For PFOA, concentrations decreased from $0.08-1.6 \mathrm{ng} / \mathrm{g} \mathrm{dw}$ in 2004 to $0.007-0.43 \mathrm{ng} / \mathrm{g} \mathrm{dw}$ in 2011, a trend that might be attributed to the voluntary phase-out by manufacturers (3M, 2011). The median concentrations of PFBS and PFHxS were $0.054 \mathrm{ng} / \mathrm{g} \mathrm{dw}$ and $0.056 \mathrm{ng} / \mathrm{g}$ dw in 2011 which are slightly higher than those measured in 2004 (median $<0.03 \mathrm{ng} / \mathrm{g}$ for PFBS and PFHxS). Whereas, PFNA and PFDA showed comparable concentrations in $2004(0.055 \mathrm{ng} / \mathrm{g} \mathrm{dw}$ for PFNA, $0.064 \mathrm{ng} / \mathrm{g} \mathrm{dw}$ for PFDA) and in 2011 ( $0.032 \mathrm{ng} / \mathrm{g} \mathrm{dw}$ for PFNA, 0.059 ng/g dw for PFDA).

\subsection{Environmental risk assessment of $P F O S$ and $P F O A$}

Environmental risk assessment (ERA) was conducted for PFOS and PFOA in German Bight utilizing risk quotient (RQ), which is defined as the ratio of measured environmental concentration (MEC) to the predicted no-effect concentration (PNEC), where RQ $<1$ indicates no potential risk. The PNEC selected in this study is derived by the equilibrium partitioning method, which has been calculated for PFOS and PFOA

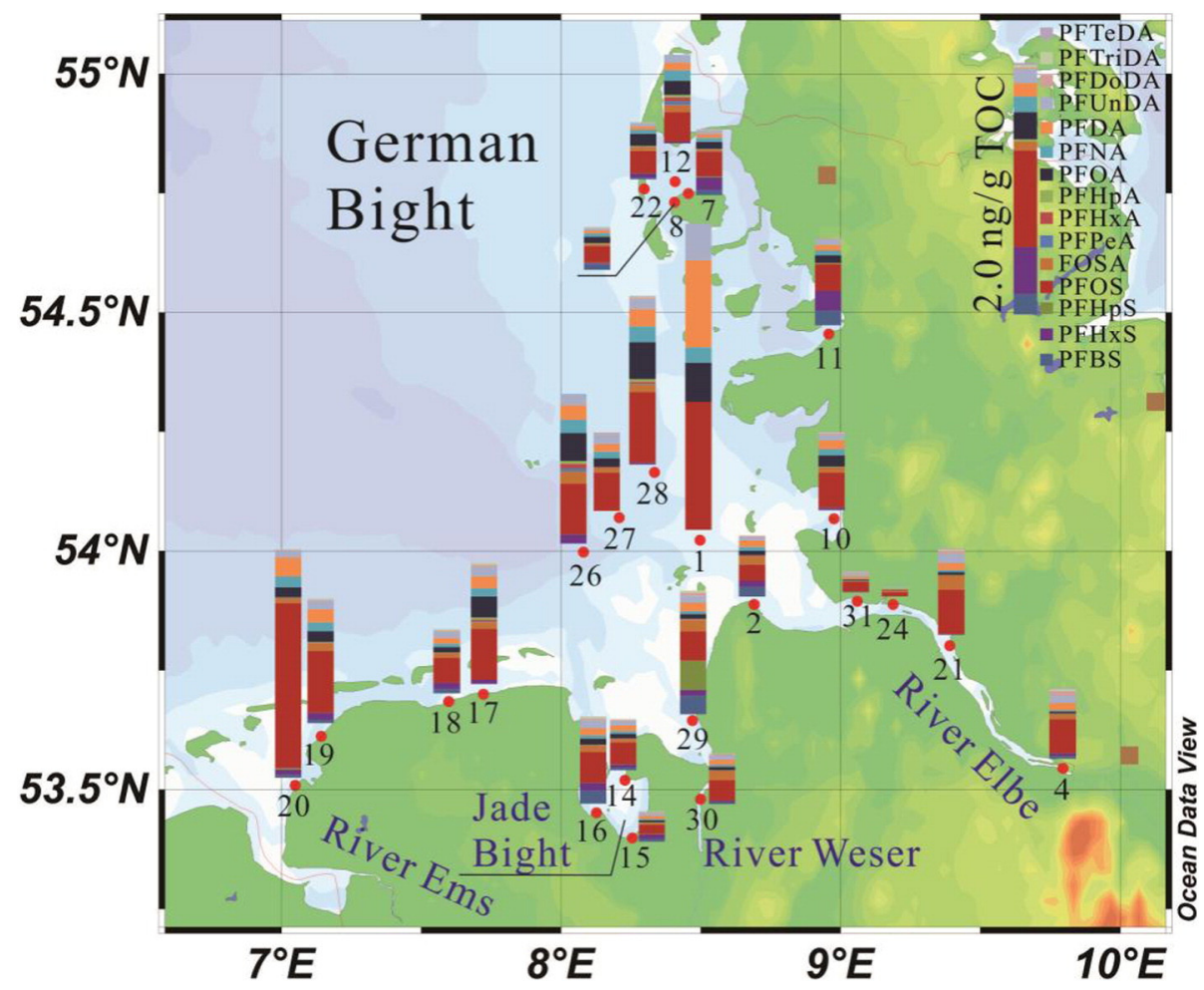

Fig. 3. TOC-normalized concentrations (ng/g dw) and spatial distribution of PFAAs in German Bight surface sediment. 
Table 2

Comparisons of PFOA and PFOS level in the German Bight and other coastal zones in the world.

\begin{tabular}{|c|c|c|c|c|}
\hline Location & Sampling year & PFOS & PFOA & Reference \\
\hline North Bohai Sea Coast, China & 2008 & nd-1.97 & nd-0.542 & Wang et al. 2011) \\
\hline Laizhou Bay, China & 2009 & $<0.03-0.06$ & $0.07-1.8$ & Zhao et al. (2013) \\
\hline Tokyo Bay, Japan & 2004 & 1.66 & 0.45 & Zushi et al. (2010) \\
\hline Ariake Sea, Japan & 2004 & $0.09-0.14$ & $0.84-1.1$ & Nakata et al. (2006) \\
\hline Savannah River Estuary, USA & 2007 & $0.3-0.8$ & nd-0.2 & Senthil et al. (2009) \\
\hline LCP Superfund Site, USA & 2006 & $0.1-0.4$ & nd -0.2 & Senthil et al. (2009) \\
\hline San Francisco Coast, USA & 2002, 2004 & nd-3.07 & nd-0.625 & Higgins et al. (2005) \\
\hline Sydney Harbor, Australia & 2009 & $0.80-6.2$ & $0-0.16$ & Thompson et al. (2011) \\
\hline Roter Main River, Germany & 2006 & $0.07-0.31$ & $0.02-0.07$ & Becker et al. (2008) \\
\hline Western Baltic Sea & 2005 & $0.03-0.67$ & $0.06-0.68$ & Theobald et al. (2012) \\
\hline German Bight & 2004 & $0.04-2.4$ & $0.08-1.6$ & Theobald et al. (2012) \\
\hline German Bight & 2011 & $0.023-5.36$ & $0.007-0.43$ & This study \\
\hline
\end{tabular}

nd: not detected.

in sediments from Laizhou Bay. The non-TOC-normalized PNECs for PFOS and PFOA were $4.9 \mathrm{ng} / \mathrm{g}$ and $86 \mathrm{ng} / \mathrm{g}$ in river sediment, and $0.49 \mathrm{ng} / \mathrm{g}$ and $8.6 \mathrm{ng} / \mathrm{g}$ in marine sediment, respectively (Zhao et al., 2013).

The RQ values calculated for the sediment samples have been shown in Fig. 4. For the river sediments, the RQ values ranged from 0.03 to 0.13 for PFOS, and from 0.0001 to 0.011 for PFOA, respectively. All the RQs were less than 1, indicating no significant risk to benthic organisms. For marine sediment, the RQs ranged from 0.05 to 11 for PFOS (mean 1.1 ), and from 0.001 to 0.05 for PFOA (mean 0.01), respectively. RQs were great than 1 in $32 \%$ of the marine sediment samples. The maximum RQ for PFOS was 11, presented in sample G20 collected near the mouth of the Ems, followed by a value of 1.6 at site G22, which is influenced by the Elbe. These results suggest that PFOS may present some level of risk to benthic organisms in the German Bight, while the risks associated with PFOA appear to be negligible, based on the relatively low RQ.

\section{Conclusions}

In surface sediments from German Bight, PFOS, PFOA, and PFDA exhibited higher concentrations than other PFAAs. PFBS did not contribute greatly to IPFAA concentrations, although it was the dominant compound in the water phase. Because of their stronger affinity to the sediment, longer-chained PFAAs $(C>10)$ were more frequently detected than shorter-chained compounds, and at the same time presented lower concentrations. At semi-enclosed coastal sites, PFAAs accumulated because of the weak exchange of water and sediment, whereas PFAAs can be transported from the estuaries to open areas due to

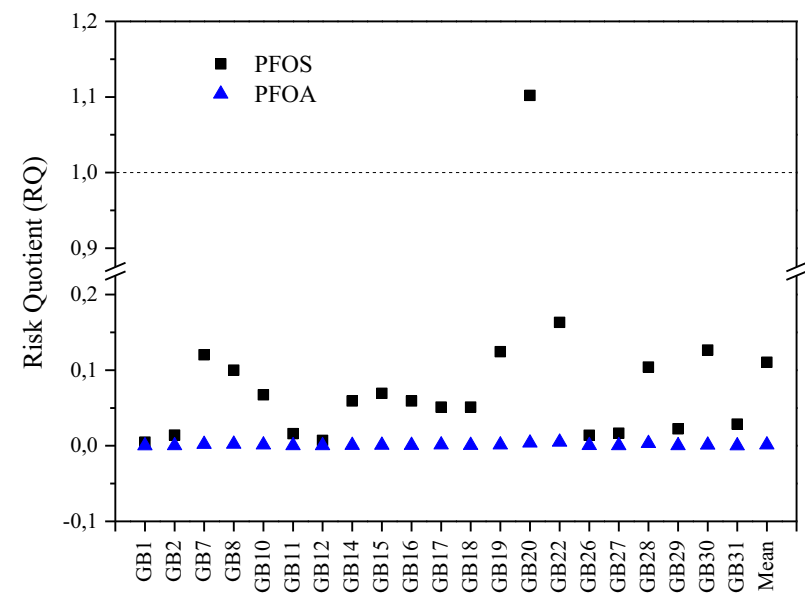

Fig. 4. Risk quotient (RQ) used for environmental risk assessment of PFOS and PFOA $(\mathrm{RQ}<1$ indicates no potential risk). currents and the homogenization effect. The distributions of PFAAs were related to the TOC content of the sediment. After normalizing the concentrations by TOC content, the PFAAs presented a different distribution. Some sites showed elevated values, which revealed the real status of contamination. Comparison with the literature showed that PFOS concentrations were higher than most other values reported worldwide, whereas PFOA presented moderate levels. Compared with previous surveys of the study area, PFOS and PFOA concentrations presented declining trends. It therefore appears that the regulation of $\mathrm{C}_{8}$-PFAAs has had positive results in German Bight, although RQ suggests that PFOS in marine sediment may represent an environmental risk to benthic organisms in German Bight.

\section{Acknowledgments}

We are grateful to the crew of the research vessel Ludwig Prandtl for their support during the sampling cruise in 2011. Steffi Quade and Renate Sturm (Helmholtz-Zentrum Geesthacht, Germany) are kindly acknowledged for organizing and assisting the sediment sampling. Zhen Zhao gratefully acknowledges the Chinese Academy of Sciences (CAS) for support via a Fellowship for Postdoctoral Research. We would like to thank the editors and reviewers for their valuable comments and suggestions.

\section{References}

3M, 2011. Re: 2011 Submission of 3M1Dyneon Under the EPA 2010/2015 PFOA Stewardship Program.

Ahrens, L., 2011. Polyfluoroalkyl compounds in the aquatic environment: a review of their occurrence and fate. J. Environ. Monit. 13, 20-31.

Ahrens, L., Barber, J.L., Xie, Z., Ebinghaus, R., 2009a. Longitudinal and latitudinal distribution of perfluoroalkyl compounds in the surface water of the Atlantic Ocean. Environ. Sci. Technol. 43, 3122-3127.

Ahrens, L., Felizeter, S., Ebinghaus, R., 2009b. Spatial distribution of polyfluoroalkyl compounds in seawater of the German Bight. Chemosphere 76, 179-184.

Ahrens, L., Plassmann, M., Xie, Z., Ebinghaus, R., 2009c. Determination of polyfluoroalkyl compounds in water and suspended particulate matter in the river Elbe and North Sea, Germany. Front. Environ. Sci. Eng. China 3, 152-170.

Ahrens, L., Taniyasu, S., Yeung, L.W.Y., Yamashita, N., Lam, P.K.S., Ebinghaus, R., 2011. Distribution of polyfluoroalkyl compounds in water, suspended particulate matter and sediment from Tokyo Bay, Japan. Chemosphere 79, 266-272.

Andersen, M.E., Butenhoff, J.L., Chang, S.-C., Farrar, D.G., Kennedy Jr., G.L., Lau, C., et al., 2008. Perfluoroalkyl acids and related chemistries - toxicokinetics and modes of action. Toxicol. Sci. 102, 3-14.

Armitage, J., Cousins, I.T., Buck, R.C., Prevedouros, K., Russell, M.H., MacLeod, M., et al., 2006. Modeling global-scale fate and transport of perfluorooctanoate emitted from direct sources. Environ. Sci. Technol. 40, 6969-6975.

Bao, J., Jin, Y., Liu, W., Ran, X., Zhang, Z., 2009. Perfluorinated compounds in sediments from the Daliao River system of northeast China. Chemosphere 77, 652-657.

Bao, J., Liu, W., Liu, L., Jin, Y., Ran, X., Zhang, Z., 2010. Perfluorinated compounds in urban river sediments from Guangzhou and Shanghai of China. Chemosphere 80, 123-130.

Bao, L.-J., Maruya, K.A., Snyder, S.A., Zeng, E.Y., 2012. China's water pollution by persistent organic pollutants. Environ. Pollut. 163, 100-108.

Becker, A.M., Gerstmann, S., Frank, H., 2008. Perfluorooctanoic acid and perfluorooctane sulfonate in the sediment of the Roter Main river, Bayreuth, Germany. Environ. Pollut. $156,818-820$. 
Benskin, J.P., Muir, D.C.G., Scott, B.F., Spencer, C., De Silva, A.O., Kylin, H., et al., 2012. Perfluoroalkyl acids in the Atlantic and Canadian Arctic Oceans. Environ. Sci. Technol. 46, 5815-5823.

Beškoski, V.P., Takemine, S., Nakano, T., Slavković Beškoski, L., Gojgić-Cvijović, G., Ilić, M., et al., 2013. Perfluorinated compounds in sediment samples from the wastewater canal of Pančevo (Serbia) industrial area. Chemosphere 91, 1408-1415.

Butt, C.M., Berger, U., Bossi, R., Tomy, G.T., 2010. Levels and trends of poly- and perfluorinated compounds in the arctic environment. Sci. Total Environ. 408, 2936-2965.

Cai, M., Zhao, Z., Yin, Z., Ahrens, L., Huang, P., Cai, M., et al., 2012. Occurrence of perfluoroalkyl compounds in surface waters from the North Pacific to the Arctic Ocean. Environ. Sci. Technol. 46, 661-668.

Campo, J., Pérez, F., Masiá, A., Picó, Y., Farré, Ml, Barceló, D., 2015. Perfluoroalkyl substance contamination of the Llobregat River ecosystem (Mediterranean area, NE Spain). Sci. Total Environ. 503-504, 48-57.

Carroll, M.L., Johnson, B.J., Henkes, G.A., McMahon, K.W., Voronkov, A., Ambrose Jr., W.G., et al., 2009. Bivalves as indicators of environmental variation and potential anthropogenic impacts in the southern Barents Sea. Mar. Pollut. Bull. 59, 193-206.

Dreyer, A., Weinberg, I., Temme, C., Ebinghaus, R., 2009. Polyfluorinated compounds in the atmosphere of the Atlantic and southern oceans: evidence for a global distribution. Environ. Sci. Technol. 43, 6507-6514.

Ellis, D.A., Martin, J.W., De Silva, A.O., Mabury, S.A., Hurley, M.D., Sulbaek Andersen, M.P. et al., 2004. Degradation of fluorotelomer alcohols: a likely atmospheric source of perfluorinated carboxylic acids. Environ. Sci. Technol. 38, 3316-3321.

EPC, 2006. Directive 2006/122/EC of the European Parliament and of the Council of 12 December 2006 Amending for the 30th Time Council Directive 76/769/EEC on the Approximation of the Laws, Regulations and Administrative Provisions of the Member States Relating to Restrictions on the Marketing and Use of Certain Dangerous Substances and Preparations (Perfluorooctane Sulfonates). European Parliament and Council, Strasbourg.

Giesy, J.P., Kannan, K., 2001. Global distribution of perfluorooctane sulfonate in wildlife. Environ. Sci. Technol. 35, 1339-1342.

Giesy, J.P., Naile, J.E., Khim, J.S., Jones, P.D., Newsted, J.L., 2010. Aquatic toxicology of perfluorinated chemicals. In: Whitacre, D.M. (Ed.), Reviews of Environmental Contamination and Toxicology. 202, pp. 1-52.

Hekster, F.M., Laane, R.W.P.M., Voogt, P., 2003. Environmental and toxicity effects of perfluoroalkylated substances. Rev. Environ. Contam. Toxicol. 179, 99-121.

Higgins, C.P., Luthy, R.G., 2006. Sorption of perfluorinated surfactants on sediments. Environ. Sci. Technol. 40, 7251-7256.

Higgins, C.P., Field, J.A., Criddle, C.S., Luthy, R.G., 2005. Quantitative determination of perfluorochemicals in sediments and domestic sludge. Environ. Sci. Technol. 39, 3946-3956.

Hloušková, V., Lanková, D., Kalachová, K., Hrádková, P., Poustka, J., Hajšlová, J., et al., 2014. Brominated flame retardants and perfluoroalkyl substances in sediments from the Czech aquatic ecosystem. Sci. Total Environ. 470-471, 407-416.

Houde, M., Martin, J.W., Letcher, R.J., Solomon, K.R., Muir, D.C.G., 2006. Biological monitoring of polyfluoroalkyl substances: a review. Environ. Sci. Technol. 40, 3463-3473.

Jahnke, A., Berger, U., Ebinghaus, R., Temme, C., 2007. Latitudinal gradient of airborne polyfluorinated alkyl substances in the marine atmosphere between Germany and South Africa (53 degrees N-33 degrees S). Environ. Sci. Technol. 41, 3055-3061.

Kampa, E., Kranz, N., Hansen, W., Madeira, N., Vorwerk, A., 2003. Public Participation in River Basin Management in Germany "From Borders to Natural Boundaries". Institute for International and European Environmental Policy.

Kannan, K., 2011. Perfluoroalkyl and polyfluoroalkyl substances: current and future perspectives. Environ. Chem. 8, 333-338.

Kannan, K., Perrotta, E., Thomas, N.J., 2006. Association between perfluorinated compounds and pathological conditions in southern sea otters. Environ. Sci. Technol. 40, 4943-4948.

Kwok, K.Y., Yamazaki, E., Yamashita, N., Taniyasu, S., Murphy, M.B., Horii, Y., et al., 2013. Transport of perfluoroalkyl substances (PFAS) from an arctic glacier to downstream locations: implications for sources. Sci. Total Environ. 447, 46-55.

Labadie, P., Chevreuil, M., 2011a. Biogeochemical dynamics of perfluorinated alkyl acids and sulfonates in the River Seine (Paris, France) under contrasting hydrological conditions. Environ. Pollut. 159, 3634-3639.

Labadie, P., Chevreuil, M., 2011b. Partitioning behaviour of perfluorinated alkyl contaminants between water, sediment and fish in the Orge River (nearby Paris, France). Environ. Pollut. 159, 391-397.

Lam, N.-H., Cho, C.-R., Lee, J.-S., Soh, H.-Y., Lee, B.-C., Lee, J.-A., et al., 2014. Perfluorinated alkyl substances in water, sediment, plankton and fish from Korean rivers and lakes: a nationwide survey. Sci. Total Environ. 491-492, 154-162.

Li, J., Del Vento, S., Schuster, J., Zhang, G., Chakraborty, P., Kobara, Y., et al., 2011. Perfluorinated compounds in the Asian atmosphere. Environ. Sci. Technol. 45, 7241-7248.

Lindstrom, A.B., Strynar, M.J., Libelo, E.L., 2011. Polyfluorinated compounds: past, present, and future. Environ. Sci. Technol. 45, 7954-7961.
Martin, J.W., Ellis, D.A., Mabury, S.A., Hurley, M.D., Wallington, T.J., 2006. Atmospheric chemistry of perfluoroalkanesulfonamides: kinetic and product studies of the $\mathrm{OH}$ radical and $\mathrm{Cl}$ atom initiated oxidation of $\mathrm{N}$-ethyl perfluorobutanesulfonamide. Environ. Sci. Technol. 40, 864-872.

Möller, A., Ahrens, L., Surm, R., Westerveld, J., van der Wielen, F., Ebinghaus, R., et al., 2010. Distribution and sources of polyfluoroalkyl substances (PFAS) in the River Rhine watershed. Environ. Pollut. 158, 3243-3250.

Naile, J.E., Khim, J.S., Wang, T., Chen, C., Luo, W., Kwon, B.-O., et al., 2010. Perfluorinated compounds in water, sediment, soil and biota from estuarine and coastal areas of Korea. Environ. Pollut. 158, 1237-1244.

Nakata, H., Kannan, K., Nasu, T., Cho, H.-S., Sinclair, E., Takemura, A., 2006. Perfluorinated contaminants in sediments and aquatic organisms collected from shallow water and tidal flat areas of the Ariake Sea, Japan: environmental fate of perfluorooctane sulfonate in aquatic ecosystems. Environ. Sci. Technol. 40, 4916-4921.

Pan, G., You, C., 2010. Sediment-water distribution of perfluorooctane sulfonate (PFOS) in Yangtze River Estuary. Environ. Pollut. 158, 1363-1367.

Pan, C.-G., Ying, G.-G., Zhao, J.-L., Liu, Y.-S., Jiang, Y.-X., Zhang, Q.-Q., 2014. Spatiotemporal distribution and mass loadings of perfluoroalkyl substances in the Yangtze River of China. Sci. Total Environ. 493, 580-587.

Paul, A.G., Jones, K.C., Sweetman, A.J., 2009. A first global production, emission, and environmental inventory for perfluorooctane sulfonate. Environ. Sci. Technol. 43 386-392.

Perra, G., Focardi, S.E., Guerranti, C., 2013. Levels and spatial distribution of perfluorinated compounds (PFCs) in superficial sediments from the marine reserves of the Tuscan Archipelago National Park (Italy). Mar. Pollut. Bull. 76, 379-382.

Prevedouros, K., Cousins, I.T., Buck, R.C., Korzeniowski, S.H., 2005. Sources, fate and transport of perfluorocarboxylates. Environ. Sci. Technol. 40, 32-44.

Prevedouros, K., Cousins, I.T., Buck, R.C., Korzeniowski, S.H., 2006. Sources, Fate and Transport of Perfluorocarboxylates. Environ. Sci. Technol. 40, 32-44.

Senthil, K.K., Zushi, Y., Masunaga, S., Gilligan, M., Pride, C., Sajwan, K.S., 2009. Perfluorinated organic contaminants in sediment and aquatic wildlife, including sharks, from Georgia, USA. Mar. Pollut. Bull. 58, 621-629.

Shoeib, M., Harner, T., Vlahos, P., 2006. Perfluorinated chemicals in the Arctic atmosphere Environ. Sci. Technol. 40, 7577-7583.

Shoeib, M., Vlahos, P., Harner, T., Peters, A., Graustein, M., Narayan, J., 2010. Survey of polyfluorinated chemicals (PFCs) in the atmosphere over the northeast Atlantic Ocean. Atmos. Environ. 44, 2887-2893.

Sinclair, E., Kannan, K., 2006. Mass loading and fate of perfluoroalkyl surfactants in wastewater treatment plants. Environ. Sci. Technol. 40, 1408-1414

Taniyasu, S., Yamashita, N., Moon, H.-B., Kwok, K.Y., Lam, P.K.S., Horii, Y., et al., 2013. Does wet precipitation represent local and regional atmospheric transportation by perfluorinated alkyl substances? Environ. Int. 55, 25-32.

Theobald, N., Caliebe, C., Gerwinski, W., Huehnerfuss, H., Lepom, P., 2012. Occurrence of perfluorinated organic acids in the North and Baltic Seas. Part 2: distribution in sediments. Environ. Sci. Pollut. Res. 19, 313-324.

Thompson, J., Roach, A., Eaglesham, G., Bartkow, M.E., Edge, K., Mueller, J.F., 2011 Perfluorinated alkyl acids in water, sediment and wildlife from Sydney Harbour and surroundings. Mar. Pollut. Bull. 62, 2869-2875.

Tomy, G.T., Tittlemier, S.A., Palace, V.P., Budakowski, W.R., Braekevelt, E., Brinkworth, L., et al., 2004. Biotransformation of N-ethyl perfluorooctanesulfonamide by rainbow trout (Oncorhynchus mykiss) liver microsomes. Environ. Sci. Technol. 38, 758-762.

USEPA, 2006. 2010/2015 PFOA Stewardship Program. U.S. EPA, Office of Pollution Prevention and Toxics, Risk Assessment Division.

Vierke, L., Staude, C., Biegel-Engler, A., Drost, W., Schulte, C., 2012. Perfluorooctanoic acid (PFOA) - main concerns and regulatory developments in Europe from an environmental point of view. Environ. Sci. Eur. 24, 16.

Wallington, T.J., Hurley, M.D., Xia, J., Wuebbles, D.J., Sillman, S., Ito, A., et al., 2006. Formation of $\mathrm{C7F} 15 \mathrm{COOH}$ (PFOA) and other perfluorocarboxylic acids during the atmospheric oxidation of 8: 2 fluorotelomer alcohol. Environ. Sci. Technol. 40, 924-930.

Wang, T., Lu, Y., Chen, C., Naile, J.E., Khim, J.S., Park, J., et al., 2011. Perfluorinated compounds in estuarine and coastal areas of north Bohai Sea, China. Mar. Pollut. Bull. 62, 1905-1914.

Yamashita, N., Kannan, K., Taniyasu, S., Horii, Y., Petrick, G., Gamo, T., 2005. A global survey of perfluorinated acids in oceans. Mar. Pollut. Bull. 51, 658-668.

Zhao, Z., Tang, J., Xie, Z., Chen, Y., Pan, X., Zhong, G., et al., 2013. Perfluoroalkyl acids (PFAAs) in riverine and coastal sediments of Laizhou Bay, North China. Sci. Total Environ. 447, 415-423.

Zhao, Z., Xie, Z., Tang, J., Sturm, R., Chen, Y., Zhang, G., et al., 2014. Seasonal variations and spatial distributions of perfluoroalkyl substances in the rivers Elbe and lower Weser and the North Sea. Chemosphere (in press).

Zushi, Y., Tamada, M., Kanai, Y., Masunaga, S., 2010. Time trends of perfluorinated compounds from the sediment core of Tokyo Bay, Japan (1950s-2004). Environ. Pollut $158,756-763$ 